\title{
COMUNICACIÓN
}

\section{Reporte parasitológico de Triakis maculata (Kner \& Steindachner, 1867) «tollo manchado» (Chondrichthyes: Selachimorpha) en el terminal pesquero Chorrillos, Lima- Perú}

\author{
Parasitological report of Triakis maculata (Kner \& Steindachner, 1867) \\ «Spotted houndshark» (Chondrichthyes: Selachimorpha) at the \\ Chorrillos fishing terminal, Lima, Perú
}

\author{
Vania Arrese-Dávila ${ }^{1,2}$, Gina Casas-Velásquez ${ }^{1}$
}

\section{Resumen}

\begin{abstract}
El objetivo del presente trabajo fue describir la fauna parasitaria de Triakis maculata (Kner y Steindachner, 1867), especie de tiburón destinado al consumo humano, para determinar la posible presencia de parásitos de implicancia en la salud pública. Se evaluaron 14 individuos T. maculata adquiridos en el Terminal Pesquero de Chorrillos, Lima, Perú. Se encontraron siete especies de parásitos, entre los que se mencionan monogeneos (Erpocotyle sp), nematodos (Proleptus sp), cestodos (Paraorygmatobothrium sp y Callitetrarhynchus sp.) y copépodos (Achtheinus sp, Caligus sp y Lernaeopoda sp). Se encontró una mayor prevalencia del cestodo Paraorygmatobothrium sp (64.3\%). Ninguno de los parásitos hallados tiene carácter zoonótico.
\end{abstract}

Palabras clave: parásitos, elasmobranquios, Triakis maculata, pesca, prevalencia

\section{Abstract}

The aim of this study was to describe the parasitic fauna of Triakis maculata (Kner y Steindachner, 1867), a species of shark sold for human consumption, to determine the possible presence of parasites of implication in public health. In total, 14 T. maculata

${ }^{1}$ Facultad de Ciencias Veterinarias y Biológicas, Universidad Científica del Sur, Lima, Perú

${ }^{2}$ E-mail: vaniadavila10@hotmail.com

Recibido: 29 de noviembre de 2020

Aceptado para publicación: 29 de junio de 2021

Publicado: 24 de agosto de 2021

CLos autores. Este artículo es publicado por la Rev Inv Vet Perú de la Facultad de Medicina Veterinaria, Universidad Nacional Mayor de San Marcos. Este es un artículo de acceso abierto, distribuido bajo los términos de la licencia Creative Commons Atribución 4.0 Internacional (CC BY 4.0) [https:// creativecommons.org/licenses/by/4.0/deed.es] que permite el uso, distribución y reproducción en cualquier medio, siempre que la obra original sea debidamente citada de su fuente original 
individuals acquired at the Chorrillos Fishing Terminal, Lima, Peru was evaluated. Seven species of parasites were found, including monogeneous (Erpocotyle sp), nematodes (Proleptus sp), cestodes (Paraorygmatobothrium sp and Callitetrarhynchus sp.) and copepods (Achtheinus sp, Caligus sp and Lernaeopoda sp). A higher prevalence of the Paraorygmatobothrium sp cestode (64.3\%) was found. None of the parasites found is zoonotic.

Key words: parasites, elasmobranchs, Triakis maculata, fishing, prevalence

\section{INTRODUCCIÓN}

Triakis maculata (Kner \& Steindachner, 1867) es un pez cartilaginoso perteneciente a la familia Triakidae (Romero et al., 2007). Su distribución por Sudamérica va desde Perú hasta el norte de Chile (Romero y Leandro, 2007). Se encuentra dentro de la lista de las especies de tiburones capturados y comercializados para el consumo humano (Romero et al., 2015). Entre los parásitos que han sido identificados en T. maculata se encuentran Acanthocheilus bicuspis (Tantaleán, 1991), Proleptus obtusus (Tantaleán y Rodríguez, 1987), Lacistorhynchus tenuis (Carvajal, 1974), Pseudolernaeopoda caudocapta, Lernaeopoda tennuis (Castro y Baeza,1986) y Paeon triakis (Castro, 2001), ubicándose en estómago, válvula espiral, intestino, cloaca y cámara branquial (Eiras et al., 2016; Luque et al., 2016).

Los copépodos (artrópodos) conforman uno de los grupos más diversos de ectoparásitos en elasmobranquios (Caira et al., 2012), pudiendo causar daños en las lamelas branquiales (Benz y Adamson, 1990) y pérdida de la capacidad visual (Benz et al., 2002), entre otros. Caira et al. (2012) mencionan que los monogeneos son el segundo grupo de ectoparásitos más diversos después de los copépodos que afectan a los elasmobranquios. Los endoparásitos pueden ser mixozoos, acantocéfalos, nematodos y la mayoría de platelmintos, aunque los monogeneos serían la excepción, ya que la mayoría suele ser ectoparásito (Caira et al., 2012). Algunos autores añaden una tercera categoría, llamada mesoparásitos, los cuales vendrían a ser aquellos que se encuentran semiexpuestos, siendo la mayoría de tipo copépodos (Castañeda, 2011; Cuyás, 2015).

Otros tiburones de la familia Triakidae, como Mustelus mento, M. whitneyi y M. dorsalis (Eiras et al., 2016; Luque et al., 2016) también se encuentran en aguas de Perú, reportándose larvas de Anisakis spp en M. dorsalis (Eiras et al., 2016). Esta especie de parásito tiene a los peces como a uno de sus hospedadores intermediarios, y a los mamíferos marinos como hospedadores definitivos; sin embargo, debido a que muchas de las especies acuáticas son destinadas para el consumo humano, el hombre actúa como un hospedador accidental de este parasito, ocasionándose la anisakidosis (Serrano-Martínez et al., 2017). Además, del Anisakis spp, otros parásitos en peces marinos de consumo humano presentan un potencial zoonótico, como los cestodos Diphyllobothrium pacificum y D. latum, que causan la difilobotriasis, aunque también puede tener un curso asintomático (Oliveira et al., 2017). Otra especie de potencial zoonótico es el acantocéfalo Corynosoma sp (Torres et al., 2014), y que, además puede infectar al perro doméstico (Naupay et al., 2019). 
La presencia de parásitos en peces no solo puede llegar a perjudicar al ser humano (parásitos zoonóticos) y a otros animales, sino que también afecta su crecimiento y supervivencia. Por otro lado, infestaciones masivas en la musculatura de los peces puede provocar una apariencia desagradable o el deterioro del pescado, siendo menos atractivo para el consumidor, afectando su valor comercial (Eiras et al., 2016).

La escasa información que se tiene acerca de parásitos en T. maculata, no permite descartar la presencia de algún agente parasitario de implicancia humana. Es por ello, que el objetivo del trabajo fue describir la fauna parasitaria de T. maculata del terminal pesquero de Chorrillos, ubicado en el departamento de Lima, Perú.

\section{Materiales y Métodos}

Se obtuvo una muestra de 14 individuos frescos de Triakis maculata (Kner \& Steindachner, 1867) (5 hembras y 9 machos) adquiridos en el Terminal Pesquero de Chorrillos en Lima, Perú, entre septiembre de 2019 y febrero de 2020. Los especímenes fueron identificados con la clave de Chirichigno y Vélez (1998). Posteriormente fueron trasladados al laboratorio de investigación de la Universidad Científica del Sur en recipientes térmicos con hielo.

En el laboratorio se registraron los datos de sexo, peso y longitud total. Se realizó la observación externa del espécimen (piel, aletas, ojos, boca, hendiduras branquiales y cavidades) siguiendo el protocolo establecido por Pavanelli et al. (1998). Para la observación interna, se realizó un corte en el área abdominal, desde el ano hasta la altura de aletas pectorales. Se realizó la observación in situ de los órganos y luego fueron retirados. Se examinó el tubo digestivo, el mesen- terio visceral y los músculos esque-léticos siguiendo el protocolo de Chero (2017).

Los parásitos extraídos fueron colocados en placas Petri para su visualización por medio de un estereoscopio y microscopio a fin de identificarlos mediante descripciones morfológicas, con base a publicaciones relacionadas a parásitos en peces (Sarmiento et al., 1999; Caira et al., 2012; Morales, 2015; Eiras et al., 2016; Luque et al., 2016; Méndez y Dorantes, 2017).

Se calculó la prevalencia $(\mathrm{P})$ y abundancia media (A) de los parásitos, según Margolis et al. (1982) y Bush et al. (1997), determinando la especie de parásito dominante, además del grado de parasitismo.

\section{Resultados}

Doce de los 14 individuos analizados presentaron una comunidad parasitaria compuesta por siete especies de parásitos localizaciones en piel, branquias, boca, cloaca, estómago y válvula espiral. Los cestodos Paraorygmatobothrium sp y Callitetrarhynchus sp y los copépodos Achtheinus sp, Caligus sp y Lernaeopoda sp fueron los grupos de mayor presencia en los especímenes (2 y 3 , respectivamente), donde el cestodo Paraorygmatobothrium sp (64.3\%) se observó como el parásito más dominante, seguido de Lernaeopoda sp (21.4\%). Asimismo, la mayor abundancia se registró con el cestodo Paraorygmatobothrium sp (5.64) y el nematodo Proleptus sp (3.50) (Cuadro 1).

Respecto a la localización de parásitos en el hospedador, se determinó una mayor prevalencia de endoparásitos $(78.6 \%, 11 / 14)$ frente a ectoparásitos $(42.9 \%, 6 / 14)$. E1 50\% de las infestaciones correspondió a monoparasitismo, mientras que $35.7 \%$ presentaron más de una especie de parásito (multiparasitismo) (Cuadro 2). 
Cuadro 1. Prevalencia y abundancia media de las especies de parásitos encontrados en 14 especímenes de Triakis maculata adquiridos en el Terminal Pesquero de Chorrillos, Lima, Perú

\begin{tabular}{|c|c|c|c|c|c|}
\hline Taxón/especie & Lugar & $\begin{array}{l}\text { Parásitos } \\
\text { (n) }\end{array}$ & $\begin{array}{l}\text { Peces } \\
\text { (n) }\end{array}$ & $\begin{array}{c}\text { Prevalencia } \\
(\%)\end{array}$ & $\begin{array}{c}\text { Abundancia } \\
\text { media }\end{array}$ \\
\hline \multicolumn{6}{|l|}{ Copepoda } \\
\hline Achtheinus sp & $\mathrm{P}$ & 2 & 2 & 14.29 & 0.14 \\
\hline Caligus sp & $\mathrm{P}$ & 2 & 1 & 7.14 & 0.14 \\
\hline Lernaeopoda $\mathrm{sp}$ & $\mathrm{C}$ & 4 & 3 & 21.43 & 0.29 \\
\hline \multicolumn{6}{|l|}{ Monogenea } \\
\hline Erpocotyle sp & $\mathrm{HB}$ & 1 & 1 & 7.14 & 0.07 \\
\hline \multicolumn{6}{|l|}{ Nematoda } \\
\hline Proleptus sp & $\mathrm{HB}, \mathrm{CB}, \mathrm{E}$ & 49 & 1 & 7.14 & 3.50 \\
\hline \multicolumn{6}{|l|}{ Cestoda } \\
\hline Paraorygmatobothrium sp & $\mathrm{VE}$ & 79 & 9 & 64.29 & 5.64 \\
\hline Callitetrarhynchus sp & $\mathrm{E}$ & 1 & 1 & 7.14 & 0.07 \\
\hline
\end{tabular}

P: Piel, C: Cloaca, HB: Hendidura branquial, CB: Cavidad bucal, E: Estómago, VE: Válvula espiral

Cuadro 2. Grado de parasitismo de especímenes de Triakis maculata obtenidos en el Terminal Pesquero de Chorrillos, Lima, Perú. $(\mathrm{n}=14)$

\begin{tabular}{lcc}
\hline \multirow{2}{*}{$\begin{array}{l}\text { Grado de } \\
\text { parasitismo }\end{array}$} & \multicolumn{2}{c}{ Total } \\
\cline { 2 - 3 } & $\mathrm{n}$ & $\%$ \\
\hline Monoparasitismo & 7 & 50.0 \\
Multiparasitismo & 5 & 35.7 \\
Ausencia & 2 & 14.3 \\
\hline Total & 14 & 100.0 \\
\hline
\end{tabular}

\section{Discusión}

En el presente estudio se observó la presencia de siete especies de parásitos en una muestra de 14 tiburones de la especie $T$. maculata. Se encontraron cuatro especies de ectoparásitos (monogéneos y copépodos) y tres especies de endoparásitos (nematodos y cestodos), siendo el grupo de cestodos quien registró un mayor número de parásitos (80), seguido de nematodos (49).

Los cestodos son adquiridos por los elasmobranquios a través de la cadena alimentaria (Benz y Bullard, 2004; Caira et al., 2012). En el presente estudio, los cestodos Callitetrarhynchus sp y Paraorygmatobothrium sp fueron hallados en el estómago y la válvula espiral, respectivamente; siendo esta última especie la de mayor prevalencia (64.3\%). Se reporta que los cestodos son encontrados con mayor frecuencia en la válvula espiral (Caira et al., 2012), así como en el estómago (Benz y Bullard, 2004). Asimismo, se reporta que las faunas más empobrecidas de cestodos se encuentran en tiburones de aguas más profundas (Caira y Jensen, 2014).

Paraorygmatobothrium sp fue el parásito más dominante $(64.3 \%)$ y abundante (5.64), encontrado en 9 de las 14 muestras 
de T. maculata. Paraorygmatobothrium sp pertenece a la familia Phyllobothriidae y ha sido reportada en la válvula espiral de distintos elasmobranquios, como Carcharhinus dussumieri (Océano Indico) (Malek et al., 2010), Prionace glauca, Alopias vulpinus (Océano Atlántico), Triakis semifasciata (Océano Pacifico) (Ruhnke, 1994), Carcharhinus leucas, Carcharhinus limbatus, Sphyrna lewini y Rhizoprionodon terraenovae (Océano Atlántico) (Méndez y Dorantes, 2017). Por otro lado, se ha observado que Paraorygmatobothrium sp llega a madurar en grandes tiburones, mayormente Carcharhiniformes (Caira y Jensen, 2014).

El parásito Callitetrarhynchus sp se encontró adherido a las paredes del estómago con una baja prevalencia (7.1\%) y abundancia (0.07). Esta especie del orden Trypanorhyncha se caracteriza por la presencia de un aparato tentacular notorio en el escólex (Campbell y Beveridge, 1994). Ha sido reportado en elasmobranquios como Prionace glauca, Sphyrna zygaena (Brasil) (Knoff et al., 2002), Mustelus canis (Brasil) (Carmona De São Clemente y Corrêa, 1989) y Mustelus mosis (Irak) (Ali, 2008).

El nematodo Proleptus sp registró una baja prevalencia $(7.1 \%)$, pero fue la segunda especie con mayor abundancia (3.50). Se caracteriza por presentar un collar cefálico en el escólex, además de presentar una cola cónica con punta redondeada (Moravec et al., 2002). Ha sido reportada en el estómago e intestino de especies de la familia Triakidae, encontrándose en el Pacifico Sur en las especies de T. maculata (Tantaleán et al., 1982) y Mustelus mento (Tantaleán y Rodríguez, 1987) participando como hospederos definitivos para Proleptus sp (Sarmiento et al., 1999)), mientras que en el Pacifico Norte se ha reportado en la especie Mustelus californicus y Mustelus henlei (Specian et al., 1975).
El copépodo con mayor prevalencia (21.4\%) y abundancia (0.29) fue Lernaeopoda sp, encontrada en la cloaca de tres individuos T. maculata. Lernaeopoda tenuis ha sido reportada para T. maculata en la costa de Chile (Castro y Baeza, 1986). Asimismo, se ha observado que algunas especies del género Lernaeopoda son relativamente específicas con respecto al hospedero, encontrándose mayormente en hospederos de la familia Triakidae y Schyliorhinidae (Dippenaar, 2020).

Achtheinus sp fue encontrado en la base de las aletas dorsales de dos individuos T. maculata, con una prevalencia de $14.3 \%$ y una abundancia de 0.14 . Este se encuentra mayormente en las aletas de elasmobranquios y con menor frecuencia en branquias y fosas nasales de tiburones como Galeorhinus galeus, Carcharias taurus, Squalus megalops (Dippenaar y Jordaan, 2015), así como en Triakis semifasciata (Izawa, 2010), observándose su gran capacidad de adhesión a la epidermis de los tiburones, a pesar de que estos presentan escamas placoideas, lo cual dificulta la fijación de ectoparásitos (Meyer y Seegers, 2012).

El copépodo Caligus sp se presentó con una prevalencia de $7.1 \%$ y una abundancia de 0.14 . Pertenece a la familia Caligidae y suele presentar un cuerpo aplanado dorsoventralmente y suborbicular, con un abdomen con 1 a 5 segmentos (Ho y Lin, 2004). Ha sido encontrado en elasmobranquios como Carcharhinus limbatus (Rodríguez-Santiago et al, 2014), Aetobatus narinari e Hypanus americanus (Rodríguez-Santiago et al., 2016).

El monogeneo Erpocotyle sp, ubicado en la hendidura branquial, pertenece a la familia Hexavothriidae. Ha sido reportado en especies Mustelus dorsalis en aguas al norte del Perú (Tantaleán y Huiza, 1994) y en Mustelus mento (Eiras et al., 2016). 
Infestaciones masivas de Erpocotyle sp en branquias de elasmobranquios pueden provocas lesiones hiperplásicas graves en las branquias, disminuyendo el flujo de agua a través de las branquias (Bullard et al., 2001).

Ninguna de las especies de parásitos encontrados en el presente trabajo tiene implicancia en la salud humana. Eiras et al. (2016) reporta la presencia de larvas de Anisakis spp en Mustelus dorsalis; sin embargo, no fueron encontradas en el presente estudio.

\section{Conclusiones}

- Se encontraron siete especies de parásitos en una muestra de 14 tiburones Triakis maculata, procedentes del Terminal Pesquero de Chorrillos: monogéneos (Erpocotyle sp), nematodos (Proleptus $\mathrm{sp)}$, cestodos (Paraorygmatobothrium sp y Callitetrarhynchus sp) y copépodos (Achtheinus sp, Caligus sp y Lernaeopoda sp).

- El parásito hallado con mayor prevalencia fue Paraorygmatobothrium sp (64.3\%).

- No se registró la presencia de parásitos de carácter zoonótico.

\section{Literatura Citada}

1. Ali A. 2008. Taxonomy of helminth parasites in some marine and freshwater fishes and the relation of some of its with their final hosts in southern of Iraq. Ph D Thesis. Irak: University of Basrah. $336 \mathrm{p}$.

2. Benz G, Adamson S. 1990. Disease caused by Nemesis robusta (Van Beneden 1851) (Eudactylinidae: Siphonostomatoida: Copepoda) infections on gill filaments of thresher sharks (Alopias vulpinus Bonnaterre, 1758), with notes on parasite ecology and life history. Can J Zool 68: 1180-1186. doi: 10.1139/z90-175
3. Benz G, Boruncinska J, Lowry L, Whiteley H. 2002. Ocular lesions associated with attachment of the copepod Ommatokoita elongata (Lernaeopodidae: Siphonostomatoida) to corneas of Pacific sleeper shark Somniosus pacificus, captures off Alaska in Prince William Sound. J Parasitol 88: 474-481. doi: 10.1645/0022-3395(2002)088[0474:OLAWAO]2.0.CO

4. Benz G, Bullard S. 2004. Metazoan parasites and associates of Chondrichthyans with emphasis on taxa harmful to captive hosts. In: Smith M, Warmolts D, Thoney D, Hueter R (eds). The elasmobranch husbandry manual: captive care of sharks, rays and their relatives. U.S.A: Special Publication of the Ohio Biological Survey. p 325-416.

5. Bullard S, Frasca S, Benz G. 2001. Gill Lesions associated with Erpocotyle tiburonis (Monogenea: Hexabothriidae) on wild and aquarium-held bonnethead sharks (Sphyrna tiburo). J Parasitol 87: 972-977. doi: 10.1645/0022-3395(2001)087[0972:GLAWET]2.0.CO;2

6. Bush A, Lafferty K, Lotz J, Shostak A. 1997. Parasitology meets ecology on its own terms: Margolis et al., revisited. J Parasitol 83: 575-583. doi:10.2307/ 3284227

7. Caira J, Healy C, Jensen K. 2012. An updated look at elasmobranchs as hosts of metazoan parasites. In: Carrier JC, Musick JA, Heithaus MR (eds). Biology of sharks and their relatives. $2^{\text {nd }}$ ed. Florida, USA: CRC Press. p 547-578.

8. Caira J, Jensen K. 2014. A digest of elasmobranch tapeworms. J Parasitol 100: 373-391. doi: 10.1645/14-516.1

9. Campbell R, Beveridge I. 1994. Order Trypanorhyncha diesing, 1863. In: Khalil LF, Jones A, Bray RA (eds). Keys to the cestode parasites of vertebrates. UK: CAB International. p 51-148.

10. Carmona De São Clemente S, Corrêa D. 1989. Trypanorhyncha from sharks of southern Brazilian coast: Eutetrarhynchus vooremi $\mathrm{sp}$. n. and two other 
species parasites of Mustelus (Pisces, Triakidae). Mem I Oswaldo Cruz 84: 475-481. doi: 10.1590/S0074-02761989000800083

11. Carvajal J. 1974. Registro de cestodos en tiburones chilenos. Rev Parasitol 60: 29-34.

12. Castro R, Baeza H. 1986. Lernaeopoda tenuis n. sp. y Pseudolernaeopoda caudocapta ng, n. sp. (Copepoda, Lernaeopodidae) parásito en Triakis maculata (Kner y Steindachner) de la costa chilena, Pacífico Sur. Syst Parasitol 8:227-233.

13. Castañeda H. 2011. Determinación de la prevalencia y periodo de reinfestación de entero-parásitos en reptiles y aves silvestres del Zoológico de Quito en Guayllabamba. Tesis de Trabajo de Titulación. Quito: Univ. de las Américas. $121 \mathrm{p}$.

14. Castro R. 2001. Paeon triakis sp. n. and new form for Lateracanthus quadripedis Kabata et Gusev, 1966 (Copepoda: Siphonostomatoida), parasite on Chilean fishes in the South Pacific. Acta Parasitol 46: 24-29.

15. Chero J. 2017. Biodiversidad de metazoos parásitos en peces de la zona marino-costera de Lima, Perú. Tesis de Maestría. Lima, Perú: Univ. Nacional Mayor de San Marcos. 116 p.

16. Chirichigno N, Vélez J. 1998. Clave para identificar los peces marinos del Perú. IMARPE. $2^{\circ}$ ed. Perú. 496 p.

17. Cuyás C. 2015. Estudio parasitológico de osteíctios de interés pesquero en $\mathrm{Ca}$ narias. Tesis Doctoral. Las Palmas de Gran Canaria: Univ. de Las Palmas de Gran Canaria. $281 \mathrm{p}$.

18. Dippenaar S, Jordaan A. 2015. How females of Achtheinus spp (Pandaridae: Siphonostomatoida) attach to their elasmobranch hosts with notes on their effects on the hosts' fins. Folia Parasit 62: 2015.005. doi: 10.14411/fp.2015.005

19. Dippenaar S. 2020. Lernaeopoda species (Lernaeopodidae: Siphonostomatoida) from South Africa with the redescription of Lernaeopoda musteli Thomson, 1890. Mar Biodivers 50: 21.

20. Eiras J, Velloso A, Pereira J. 2016. Parasitos de peixes marinhos da América do Sul. Ed da Furg. 445 p.

21. Ho J, Lin C. 2004. Sea liceo of Taiwan. The Sueichan Press. Taiwan. p 1-388.

22. Izawa K. 2010. Resurrection of the parasitic copepod genus Achtheinus Wilson,1908 (Siphonostomatoida, Pandaridae), with redescription of $A$. oblongus Wilson, 1908. A. dentatus Wilson, 1911, and A. pinguis Wilson, 1912 based on museum collections. Crustaceana 83: 971-995.

23. Knoff M, Carmona De São Clemente S, Magalhães R, Corrêa D. 2002. Prevalência e intensidade de infecção de cestóides Trypanorhyncha de elasmobrânquios nos estados do Paraná e Santa Catarina, Brasil. Parasitol Latinoam 57: 149-157.

24. Luque J, Cruces C, Chero J, Paschoal F, Alves P, Silva A, Iannacone J. 2016. Lista de verificación de los metazoos parásitos de peces de Perú. Neotrop Helminthol 10: 301-375.

25. Malek M, Caira J, Haseli M. 2010. Two new species of Paraorgmatobothrium Ruhnke, 1994. (Cestoda: Tetraphyllidea) from the carcharhinid shark Carcharhinus cf. dussumieri (Muller \& Henle) in the Persian Gulf. Syst Parasitol 76:59-68.

26. Margolis L, Esch G, Holmes J, Kuris A, Schad G 1982. The use of ecological terms in parasitological terms (report of an ad hoc Committee of the American Society of Parasitologists) Syst Parasitol 68: 131-133.

27. Méndez O, Dorantes M. 2017. Helmintos parásitos intestinales de tiburones en la costa central del estado de Veracruz, México. Ciencia Pesquera 25: 51-61.

28. Meyer W, Seegers U. 2012. Basics of skin structure and function in elasmobranchs: a review. J Fish Biol 80: 19401967. 
29. Morales J. 2015. Taxonomía y ecología de los parásitos helmintos de los eufáusidos y de sus depredadores en el Golfo de California para inferir sus ciclos de vida. Tesis Doctoral. México: Instituto Politécnico Nacional- CICI-MAR. 203 p.

30. Moravec F, Van As J, Dyková I. 2002. Proleptus obtusus Dujardin, 1845 (Nematoda: Physalopteridae) from the puffadder shyshark Haploblepharus edwardsii (Scyliorhinidae) from off South Africa. Syst Parasitol 53: 169-173

31. Naupay A, Castro J, Rojas V, Suarez D. 2019. Helmintos gastrointestinales de Otaria flavescens Shaw 1800 (Mammalia: Otariidae) león marino sudamericano de la costa central del Perú. Neotrop Helminthol 13: 317-333.

32. Oliveira S, Nunes E, De Sousa A, Marques F, Ramos I, Da Silva M, De Oliveira T, et al. 2017. Estudo do número de casos de difilobotriase no Brasil. J Biol Pharm Agric Manag 13: 29-38.

33. Pavanelli G, Eiras J. 1998. Patologia de peixes em piscicultura no Brasil: situaçao actual e perspectivas futuras. En: V Encontro Brasileiro de Patologistas de Organismos Aquaticos. Maringá, Brasil.

34. Rodriguez-Santiago M, Gómez S, Cacho-Torres D, Ovalles-Cruz H, LópezGarcía K, Bustamante-Avedaño A, Pérez-Jiménez J. 2014. Fauna parasitaria de cinco especies de tiburones (Pisces: Elasmobranchia) en el sur del Golfo de México. En: IV Encuentro Colombiano sobre Condrictios. Medellín.

35. Rodríguez-Santiago M, MoralesSerna F, Gómez S, Grano-Maldonado M. 2016. New records of parasitic copepods (Copepoda: Pandaride, Eudactylinidae, Caligidae) on elasmobranchs (Chondrichthyes) in the Gulf of Mexico. Ciencia Pesquera 24:15-21.

36. Romero M, Leandro L. 2007. Triakis maculata. The IUCN Red List of Threatened Species 2007: e.T63130A12620764. [Internet]. Disponible en: http://dx.doi.org/10.2305/IUCN.UK.2007.RLTS.T63130A12620764.en
37. Romero M, Alcántara P, Verde K. 2015. Guía de campo para la determinación de tiburones en la pesca artesanal del Perú. Lima: Instituto del Mar del Perú. $16 \mathrm{p}$.

38. Ruhnke T. 1994. Paraorygmatobothrium barberi n.g., n. sp. (Cestoda: Tetraphyllidea), with amended descriptions of two species transferred to the genus. Syst Parasitol 28 65-79.

39. Sarmiento L, Tantaleán M, Huiza A. 1999. Nematodos parásitos del hombre y de los animales en el Perú. Rev Per Parasitol 14: 9-65.

40. Serrano-Martínez E, Quispe M, Hinostroza E, Plasencia L. 2017. Detección de parásitos en peces marinos destinados al consumo humano en Lima Metropolitana. Rev Inv Vet Perú 28: 160168. doi: 10.15381/rivep.v28i1.12935

41. Specian R, Ubelaker J, Dailey M. 1975. Neoleptus gen. n. and a revision of the genus Proleptus Dujardin, 1845. P Helm Soc Wash 42: 14-21.

42. Tantaleán M, Carvajal G, Martínez R, Huiza A. 1982. Helmintos parásitos de peces marinos de la costa peruana. NCTL. Serie de Divulgación Científica. Lima. $40 \mathrm{p}$.

43. Tantaleán M, Rodríguez J. 1987. Nuevos registros de helmintos parásitos de peces elasmobranquios de las costas del Perú. Rev Biol Trop 35: 167-168.

44. Tantaleán M. 1991. Nuevos helmintos parásitos en peces elasmobranquios de la costa peruana. Bol Lima 13: 25-28.

45. Tantaleán M, Huiza A. 1994. Sinopsis de los parásitos de peces marinos de la costa peruana. Biotempo 1: 53-101.

46. Torres P, Puga S, Castillo L, Lamilla J, Miranda, J. 2014. Helmintos, myxozoos y microsporidios en músculos de peces comercializados frescos y su importancia como riesgo potencial para la salud humana en la ciudad de Valdivia, Chile. Arch Med Vet 46: 83-92. doi: 10.4067/S0301-732X2014000100012 